\title{
Motivación y actitudes para el aprendizaje de lenguas de dos colectivos de estudiantes universi- tarios: futuros maestros de lenguas extranjeras y futuros enfermeros $y$ fisioterapeutas ${ }^{1}$
}

\author{
Elisabet SARAVIA \\ Universitat Ramon Llull \\ Mercè Bernaus \\ Facultad de Ciencias de la Educación \\ Universitat Autònoma de Barcelona
}

Escuela Universitaria de Enfermeria, Fisioterapia y Nutrición Blanquerna

Recibido: 4 julio 2007 / Aceptado: 10 noviembre 2007

ISSN: $1697-7467$

\begin{abstract}
RESUMEN: El objetivo de esta investigación es el de analizar la motivación y las actitudes hacia las lenguas en un contexto multilingüe. La muestra de este estudio cuenta con 178 estudiantes pertenecientes a dos universidades una en el ámbito de la educación y la otra en el ámbito de la salud. Como instrumento de investigación se ha utilizado un cuestionario que incluye entre otros algunos ítems sobre actitudes y motivación en el aprendizaje del castellano, catalán, inglés y francés. La autoavaluación de los estudiantes en estas cuatro lenguas correlaciona con sus actitudes y motivación hacia las mismas. Los resultados apoyan los obtenidos por otros estudios, que muestran una relación estrecha entre las actitudes, la motivación y el nivel de competencia lingüística. Además, se han llevado a cabo ANOVAS para analizar la variancia entre los diferentes grupos de estudiantes.
\end{abstract}

Palabras clave: Motivación, actitudes, aprendizaje de lenguas, multilingüismo.

\begin{abstract}
The purpose of this investigation is to analyse motivation and attitudes, towards languages in a multilingual context. It draws on a study of 178 language students in two universities one on the field of Teaching Education and the other one in the field of Health Care. A multiple-choice questionnaire was created to gather the data. It included among others some items on Attitudes and Motivation toward learning Spanish, Catalan, English and French. The students' self-assessment on those 4 languages was correlated with their answers related to attitudes and motivation toward those languages. Besides, ANOVAs were run to analyse the variability between and among different groups of students. The results supported the findings reported by other studies, showing a close relationship between attitudes, motivation and language achievement.
\end{abstract}

Key words: Motivation, attitudes, language learning, multilinguism.

1. Este artículo da a conocer parte de los resultados obtenidos en la tesis doctoral de Saravia 2004. 


\section{INTRODUCCIÓN}

El objetivo de este estudio ha sido el de observar la influencia que los factores individuales pueden tener en el aprendizaje de lenguas segundas o extranjeras en estudiantes universitarios. Este trabajo es una nueva aportación a la investigación en docencia universitaria, especialmente en el ámbito de lenguas, porque incluye estudiantes procedentes de centros académicos tan diversos como la enfermería y la fisioterapia, y las ciencias de la educación.

En segundo lugar, el estudio relaciona las cuatro lenguas de instrucción (castellano, catalán, inglés y francés) de los estudiantes con factores individuales. El catalán y el castellano se han considerado L1 o L2 según si los estudiantes se consideraban catalano-parlantes o castellano-parlantes, y el inglés y el francés LE. En pocos estudios se incluyen resultados que relacionen cuatro lenguas diferentes (catalán, castellano, ingles y francés) con la motivación y las actitudes (uno de ellos por ejemplo es el de Lasagabaster 2003). Nuestro contexto social, cada vez más complejo y cambiante, nos ha hecho considerar esta realidad multilingüe y multicultural.

En el marco bibliográfico sobre estudios similares, se comenta cómo hay trabajos que indagan en las motivaciones, las actitudes, la ansiedad, las estrategias de aprendizaje, la personalidad, y la competencia lingüística a la hora de estudiar lenguas tanto en la enseñanza secundaria como en la enseñanza universitaria. Ahora bien, la mayoría lo hacen tratando estos aspectos de forma aislada. Quizá cabe en este punto citar a modo de excepción el estudio de Madrid (1999), pues investiga la enseñanza de LE en primaria y secundaria tratando simultáneamente aspectos motivacionales y actitudinales hacia la clase, los materiales didácticos, los hablantes, la personalidad, las orientaciones, así como la figura del profesor. En este estudio hemos recogido estos trabajos y los hemos reunido. No conocemos ningún otro estudio que haya correlacionado la percepción de los estudiantes de su propio aprendizaje, con las motivaciones y las actitudes, las preferencias metodológicas de enseñanza en el aula y el uso de las estrategias, la personalidad y los factores contextuales de los encuestados. En este sentido, creemos que se trata de una investigación muy completa, y única.

\section{Marco Teórico}

En consideración al concepto de motivación y actitudes del que hemos partido, desde una perspectiva educativa se reconoce ampliamente que tanto la una como la otra son factores esenciales para un aprendizaje efectivo. Principalmente, porque sin motivación no hay aprendizaje y porque las actitudes apoyan dicha motivación. Situándonos en el marco de la psicología educativa y social, hemos seguido a Gardner y su modelo socioeducativo de la adquisición de segundas lenguas (Gardner 1985). La teoría motivacional de Gardner incluye una dimensión educacional y trata la evaluación del estudiante en la situación de aprendizaje en el aula y fuera del aula. Asume que el contexto social determina las actitudes de los aprendices. Por un lado, el grado de motivación de un alumno por el aprendizaje viene condicionado por lo que quiera conseguir haciendo una actividad concreta. Así pues, la motivación está dotada de una fuerza conductora o interés (el esfuerzo, el deseo y el afecto del individuo), 
que comporta un proceso continuo, dinámico y cambiante, ya que evoluciona según los intereses y las prioridades de la persona. Si la motivación resulta ser una variable compleja por su multiplicidad, por otro lado, tampoco resulta fácil precisar qué es la actitud. En la actitud confluyen la individualidad, la personalidad, el medio social, y los valores del estudiante; factores que una vez interiorizados resultan en una valoración que el alumno hace tanto de la lengua, de la comunidad de hablantes y de la cultura que aprende como de las propias. Una actitud positiva hacia la LE, los hablantes, y la cultura influirá en una mayor motivación del alumno por el aprendizaje, una actitud negativa lo hará más difícil.

En definitiva, el modelo socioeducativo de la adquisición de segundas lenguas (Gardner 1985) es un modelo dinámico en el que se relacionan las actitudes y la motivación con la adquisición de la lengua. Para el estudio de la motivación y las actitudes hemos seguido el test de motivación que el mismo autor y sus colegas han desarrollado, más conocido como la Batería de Tests de Actitud y Motivación (AMTB). Desde la elaboración del AMTB en 1985 hasta la actualidad, la investigación de Gardner se ha dirigido principalmente a experimentar, modificar y adaptar este test a diferentes situaciones lingüísticas. Los resultados de estas investigaciones, han sido expuestos y difundidos por el propio Gardner en publicaciones tanto de forma individual como en colaboración con otros investigadores. Desde su elaboración, el modelo socioeducativo y el AMTB de Gardner han sido un punto de referencia para los investigadores, así como el instrumento de medida de motivación y actitudes más validado hasta hoy. En este estudio, se han seguido parcialmente los pasos de Gardner (1985), aunque haciendo una adaptación de los constructos por él propuestos en sus posteriores modificaciones. La selección de estos constructos pertenece a un estudio anterior realizado por Saravia en el 2002.

La investigación que nos ocupa si bien ha incluido una recopilación de propuestas bibliográficas ya clásicas, que han marcado la evolución del aprendizaje en segundas lenguas (Costa y McCrae, Oxford, Gardner), estudios sobre motivación y actitudes hacia el aprendizaje de lenguas desde la perspectiva de la psicología educativa y social, tampoco ha olvidado la bibliografía más actual sobre el tema (Masgoret y Gardner 2003, y Dörnyei 2003). Algunas de las lecturas bibliográficas están basadas tanto en la motivación en la enseñanza secundaria (Ramage 1990; Alonso Tapia 1999; Velasco y Bernaus 1999; Madrid 1999) de donde mayoritariamente provienen los estudiantes encuestados, como en el ámbito universitario (Ely 1986; Coleman 1998 y 2002; Knowles 2001, Lasagabaster 2003). Otras referencias se basan en la motivación por el aprendizaje de segundas lenguas y/o lenguas extranjeras (Yalden 1987; Lightbown \& Spada 1993; Dörnyei 2003).

En cuanto a los estudios más representativos similares a nuestra investigación, un grupo incluye estudios sobre las motivaciones a la hora de estudiar lenguas en la enseñanza universitaria. Son estudios cuantitativos con cuestionarios sobre motivaciones y actitudes, que han utilizado una escala de Likert al igual que este trabajo. Norris (2001) estudia el papel que juega el modelo socioeducativo de Gardner y la motivación en la adquisición de una segunda lengua en la enseñanza del inglés en Japón. Norris (2001) concluye su estudio asegurando que no importa cual sea la motivación subyacente para estudiar una segunda lengua, lo que no se puede discutir es la importancia de la variable motivacional al examinar la adquisición exitosa en una segunda lengua.

Hay que aludir también a Coleman $(1995 a ; 1998 ; 2002)$ que lleva a cabo un estudio piloto descriptivo, de gran envergadura, con unos 3.000 alumnos de LE del Reino Unido y 
otros países europeos. El autor intenta comprobar los conocimientos y la efectividad de la enseñanza de lenguas en la educación superior. El cuestionario quiere descubrir cuestiones referentes al papel de la motivación y las actitudes en el aprendizaje de lenguas en la universidad; incorpora igualmente un test de competencia lingüística, así como informaciones sobre datos biográficos, de contexto de aprendizaje de lenguas, de estancias en el extranjero, estrategias y conocimientos gramaticales.

Con características similares al trabajo de Coleman (1995a; 1998; 2002) contamos con la investigación de Lasagabaster (2003). El autor presenta un estudio de campo con 1087 estudiantes del País Vasco (CAV), en el que utiliza un cuestionario, basado en parámetros de multilingüismo. Su objetivo es analizar la situación de trilingüismo en la enseñanza vasca, las actitudes hacia la lengua minoritaria, la mayoritaria y la extranjera en relación con la competencia lingüística en lengua inglesa.

Otros estudios que han tratado el tema del aprendizaje de lenguas desde el punto de vista universitario son el de Masgoret, Bernaus y Gardner (2000), y el de Linde (2001). En primer lugar, Masgoret, Bernaus y Gardner (2000) llevan a cabo un trabajo sobre la adaptación cultural de unos monitores de inglés en España, mediante un cuestionario presentado a 127 universitarios del RU (39,3\% del ámbito educativo). En segundo lugar, Linde (2001) investiga las actitudes hacia la evaluación de 200 estudiantes universitarios de filología inglesa (de formación parecida a los de la FCE-UAB) de la Universidad de Granada. Su investigación se basa en un cuestionario que pretende ofrecer puntos de reflexión sobre los exámenes y los procesos de evaluación.

Igualmente, cabe mencionar los estudios que tratan de la enseñanza de lenguas adaptada a las necesidades lingüísticas y culturales de los estudiantes. En este sentido se han incluido artículos que hacen propuestas didácticas innovadoras dentro de un marco académico universitario muy parecido al de la enfermería y la fisioterapia (Martyn 2000; Frank 2000; Lepetit y Cichock 2002; Duff 2002); y al de ciencias de la educación (Linde 2001).

El artículo de Lepetit y Cichock (2002) presenta el análisis preliminar de una encuesta sobre las necesidades y aspiraciones en el aprendizaje de LE por parte de estudiantes de la profesión sanitaria en su ejercicio profesional, a raíz de algunos cambios demográficos recientes en los EE.UU. (al norte de Carolina del Sur). Las necesidades más importantes identificadas por los estudiantes de Salud se centran en las habilidades comunicativas orales, seguidas de las habilidades comunicativas escritas. También destaca la gran importancia que se otorga al conocimiento de la cultura de la lengua meta. Por eso, se pide que el contexto de aprendizaje se sitúe en un entorno que ponga en contacto los alumnos con hablantes nativos. Las necesidades descritas por Lepetit y Cichock (2002) reflejan un entorno cada vez más consciente de la existencia de un contexto laboral sanitario multilingüe y global.

Paralelamente, Martyn (2000) ha tratado el tema del aprendizaje universitario de lenguas con propósitos específicos, dentro del campo sanitario, negociando los contenidos de los programas de estudios. La autora (2000) relaciona la motivación de los estudiantes con sus estudios, y con su compromiso por la profesionalización de la enfermería en Pakistán. De hecho, Martyn (2000) constata que, fruto de la negociación, los alumnos se esfuerzan por conseguir unos resultados excelentes, no sólo para conseguir los requerimientos del curso, sino también para provocar un impacto positivo, tanto personal como profesional, en su comunidad: “...students aimed at excellence, not simply to meet course requirements, but also to make a positive impact, both personally and professionally, on their community" (Martyn, 2000:159). 
Otro trabajo coincidente con los precedentes es el de Frank (2000). En él, la autora se propone determinar los problemas de lengua y de comunicación entre el profesorado del Programa de Estudios en Ciencias de la Salud (SHP) y sus pacientes, estudiantes internacionales. Una de las fuentes para obtener los datos de análisis han sido 123 cuestionarios cumplimentados por estudiantes internacionales al acabar sus estudios de SHP, y 100 cuestionarios que han respondido los miembros de la plantilla del programa SHP. Los resultados demuestran que, tanto estudiantes como profesores, están de acuerdo en que hay problemas de comunicación en diferentes ámbitos, a causa de los diferentes procedimientos y expectativas culturales. El origen del problema ha sido atribuir algunos fracasos conversacionales a la mala educación, más que a la falta de conocimiento pragmático por parte del estudiante. Las necesidades descubiertas en este estudio afectan al profesorado médico, los estudiantes y los educadores de lengua inglesa, y son requerimientos ineludibles si se quiere ofrecer una atención médica de alta calidad.

Actualmente, con la globalización del entorno, se observa cómo las necesidades comunicativas no atienden exclusivamente propósitos específicos (por ejemplo médicos), sino también aspectos de interculturalidad y de sensibilidad cultural (Frank 2000). En consecuencia, el aprendizaje de lenguas, sea específico (Duff 2002) o no, se sitúa en contextos multilingües (Levine 2003; Lasagabaster 2003) y multiculturales (Bernaus, Masgoret, Gardner y Reyes 2004). Así, el estudio que nos ocupa relaciona las cuatro lenguas de instrucción (castellano, catalán, inglés y francés) de los estudiantes con factores individuales, con la motivación y las actitudes.

\section{Hipótesis de Investigación}

Dos de las hipótesis planteadas, a lo largo de la investigación, han querido verificar si nuestra muestra coincide con los resultados precedentes.

Hemos visto cómo el mundo educativo acepta ampliamente que la motivación y las actitudes son factores afectivos claves para un aprendizaje efectivo. En este trabajo nos propusimos determinar la fuerza de esta motivación, en los resultados de aprendizaje lingüístico, de una manera general con todos los sujetos, y de una manera diferenciada entre grupos de aprendices. De aquí surgen las hipótesis 1 y 2: La primera se centra en la motivación, las actitudes y el aprendizaje de lenguas y queda formulada de la siguiente manera:

HIPÓTESIS 1: La motivación y las actitudes correlacionan positivamente con el aprendizaje de lenguas.

La segunda hipótesis indaga sobre las diferencias en la motivación, las actitudes y el aprendizaje entre los dos colectivos de estudiantes universitarios. Se concreta así:

HIPÓTESIS 2: Los estudiantes de la Especialidad de Maestro en Lenguas Extranjeras (inglés) alcanzarán grados de motivación y actitudes más altos en el aprendizaje de lenguas, que los estudiantes de Enfermería y Fisioterapia. 


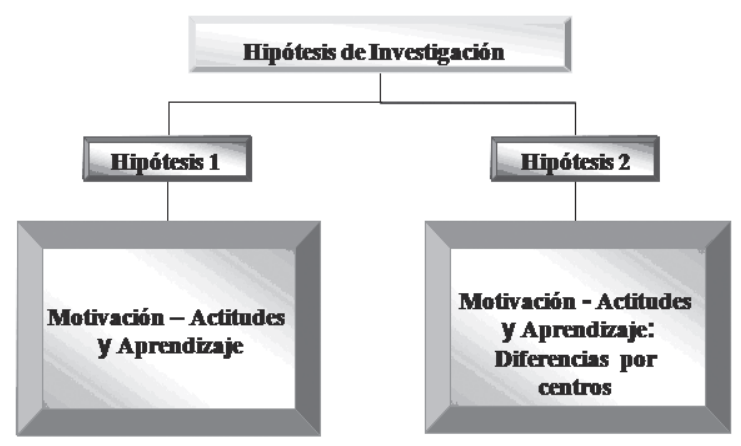

Fig.1: Hipótesis de Investigación.

\section{Metodología}

\subsection{Los Participantes y el Instrumento de Investigación}

Los Participantes en este estudio son 178 estudiantes procedentes de dos centros universitarios. Por una parte 88 estudiantes de los tres cursos de la Diplomatura de Maestro en Lenguas Extranjeras (Inglés) de la Facultad de Ciencias de la Educación, de la Universidad Autónoma de Barcelona, y por el otro, 90 estudiantes de las Diplomaturas de Enfermería y Fisioterapia, de la Escuela Universitaria de Enfermería y Fisioterapia Blanquerna, de la Universidad Ramon Llull. En cuanto a los alumnos de Enfermería y Fisioterapia, si bien, todos ellos siguen unos estudios de carácter científico, su plan académico incluye algunas asignaturas humanísticas optativas entre las cuales figuran el catalán, el francés o el inglés.

El instrumento de investigación utilizado ha sido un Cuestionario (Saravia, 2002). Éste fue elaborado por parte de un equipo de profesores, que se valió de su experiencia docente y de la discusión conjunta del grupo sobre obras de referencia para establecer sus enunciados. En primer lugar, se enmarca y explica el contexto sociocultural de los estudiantes mediante datos demográficos. A continuación, se han establecido los constructos motivacionales, actitudinales, metodológicos, de estrategias y personalidad para poderlos relacionar con la competencia lingüística en las cuatro lenguas.

Como ya hemos comentado, en el ámbito de la motivación y las actitudes, el cuestionario ha seguido parcialmente los pasos de Gardner (1985) y ha hecho una adaptación de los constructos por él propuestos en el AMTB. Los constructos incluidos en el cuestionario son los que a continuación se exponen: orientación instrumental, orientación integrativa, actitudes hacia los hablantes, actitudes hacia el aprendizaje de lenguas en el aula, intensidad motivacional, ansiedad. Igualmente, se ha introducido un nuevo constructo, la Conciencia lingüística y cultural con el interés de evaluar la conciencia de nuestros estudiantes respecto a la cultura y la lengua que aprenden, como transmisoras de unos valores socioculturales propios de una comunidad.

El cuestionario en su presentación formal a los estudiantes consta de cuatro partes (ver apéndice para el apartado de motivación y actitudes - sección 2): 
En la primera parte, los encuestados han contestado con datos precisos o marcando con una $\mathrm{X}$ cuestiones demográficas sobre su entorno familiar y social. A continuación, han evaluado numéricamente (de 1 a 10) su competencia lingüística en diferentes lenguas en las cuatro destrezas: comprensión lectora y auditiva, expresión oral y escrita (receptivas y productivas).

El propósito de la segunda parte es conocer las motivaciones y las actitudes del alumnado. Los enunciados de esta parte se han mezclado al azar para no dar pistas a los estudiantes de qué constructos se valorarían. Se ha procedido a la diversificación y gradación de las respuestas para favorecer la variancia, siguiendo una escala de valoración del 1 al 5 (totalmente en desacuerdo $=1$, bastante en desacuerdo $=2$, indiferente $=3$, bastante de acuerdo $=4$, y totalmente de acuerdo $=5$ )

Recordemos que este cuestionario incluye también una tercera y cuarta parte de las cuales no hablaremos en este artículo. En la tercera parte, se han propuesto diferentes actividades en el aula que se pueden enmarcar en las dos tendencias metodológicas de enseñanza en el aula. También se han expuesto diferentes estrategias de aprendizaje. En la última parte, se ha incluido únicamente el constructo de la personalidad.

\subsection{La Recogida de Datos y la Introducción de los mismos}

En cuanto a la recogida de datos, se pidió a los estudiantes que respondieran los 112 enunciados del cuestionario. Dentro del mismo cuestionario se ha incluido un ejemplo para ilustrar la tarea. El tiempo concedido en un principio ha sido de 15 minutos pero finalmente ha sido de 20 minutos, ya que los mismos estudiantes así lo pidieron.

La introducción y el análisis de los datos se han hecho utilizando el paquete estadístico SPSS (Statistical Package for the Social Sciences) para Windows, versión 10.0. Al utilizar el paquete estadístico SPSS, los 112 ítems del cuestionario se han agrupado en constructos. ${ }^{2}$

\section{Resultados}

A continuación, como parte del estudio empírico, hemos analizado los datos que nos llevan a resolver dos de las hipótesis de investigación planteadas de la siguiente manera:

HIPÓTESIS 1: La motivación y las actitudes correlacionan positivamente con el aprendizaje de lenguas.

Globalmente, se han observado correlaciones positivas y significativas entre las autoevaluaciones de castellano, francés e inglés con la actitud hacia el aprendizaje de la lengua, así como la intensidad motivacional por el aprendizaje de las mismas. También hay una correlación positiva y significativa entre las autoevaluaciones de lenguas extranjeras y la conciencia lingüística y cultural. Con estos resultados se puede afirmar que la primera hipótesis se cumple. 


\begin{tabular}{|c|c|c|c|c|c|}
\hline \multirow{2}{*}{ C } & Autuevaluaciones & $\begin{array}{l}\text { Autnevsil } \\
\text { Catalin }\end{array}$ & $\begin{array}{l}\text { Autoeval } \\
\text { Casteillano }\end{array}$ & $\begin{array}{l}\text { Autneval } \\
\text { Francés }\end{array}$ & $\begin{array}{l}\text { Autneval. } \\
\text { Ingless }\end{array}$ \\
\hline & Oricintación instrumental & & & & \\
\hline o & Actitud hacin el spreandiraje & & $240: \pm$ & $3^{313} \pm t$ &, $271^{ \pm \pm}$ \\
\hline $\begin{array}{r}\mathbf{R} \\
\mathbf{R}\end{array}$ & Intensidad motivacional & &, $178 \pm=$ & $.171^{*}$ & $187^{2}$ \\
\hline $\mathrm{F}$ & Conciencia lingüística & & &, $22 \sigma^{ \pm \pm}$ &, $162^{*}$ \\
\hline$\Delta$ & Actitud hacin los hablantes & & & & \\
\hline C & Orientación in tegrantiva & & & & \\
\hline $\begin{array}{l}\mathrm{O} \\
\mathrm{N}\end{array}$ & Ansiodnd & & & & ${ }_{3} 152^{ \pm}$ \\
\hline E & Autoevaluancióncatalín & &, $290^{ \pm \pm}$ & &, $230^{* \pm}$ \\
\hline S & Autoevaluarióncastellano & $29 \sigma^{ \pm \pm}$ & &, $249^{ \pm \pm}$ & $379^{ \pm \pm}$ \\
\hline & Autocvaluación firancés & &, $249 \pm t$ & &, $436^{ \pm \pm}$ \\
\hline & Autoevaluacióningglés & $230^{ \pm \pm}$ & $379^{ \pm \pm}$ &, $436^{ \pm \pm}$ & \\
\hline
\end{tabular}

Fig. 2: Correlaciones Autoevaluaciones.

Nuestro interés ha sido también el de detectar si había diferencias por escuelas, por lengua materna y autoevaluaciones. En concreto, los alumnos de Ciencias de la Educación disponen de una orientación instrumental, una orientación integrativa, una intensidad motivacional y una conciencia lingüística hacia las lenguas más marcadamente alta; y como hemos visto al presentar los resultados de las anovas, son precisamente ellos mismos los que se autoevalúan mejor en cuanto a su competencia lingüística en catalán, francés e inglés, en comparación con los futuros enfermeros o fisioterapeutas, lo cual parece lógico si pensamos en la orientación profesional de los primeros. Es por ello, también, que, manifiestan un grado de ansiedad más elevado que los de Enfermería y Fisioterapia. Posiblemente porque para sus estudios, las lenguas son materias con un peso más específico.

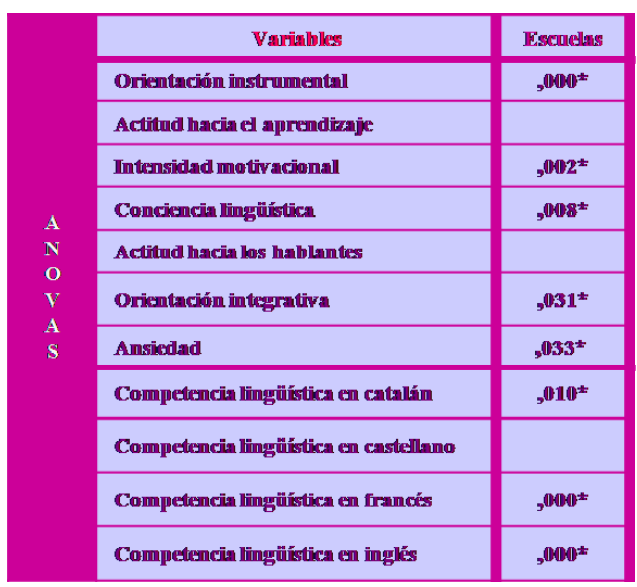

Fig.3: Anovas por Escuelas. 
Igualmente, cuanto más alta es la calificación que se asignan en lengua castellana, más positiva será su actitud hacia el aprendizaje de la lengua (castellana) y su intensidad motivacional. Al contrario, los que solamente se evalúan con un aprobado en castellano son los menos motivados por aprender lenguas. En lo referente a la lengua inglesa, a medida que mejora su nivel de lengua, más positiva es su actitud hacia el aprendizaje de la misma. Los que manifiestan tener un nivel insuficiente de inglés son los que muestran unas actitudes de aprendizaje menos positivas, son los menos conscientes del valor lingüístico y cultural de las lenguas; y son también los más ansiosos. De hecho, los dos niveles más bajos de inglés corresponden a los estudiantes más ansiosos y, en cambio, conforme mejora su nivel de lengua inglesa se reduce el grado de ansiedad. En este caso, la ansiedad elevada actúa como factor negativo en el aprendizaje. Estos resultados confirman una vez más la hipótesis de que la motivación y las actitudes muestran una correlación positiva con el aprendizaje de lenguas (ver Fig.2).

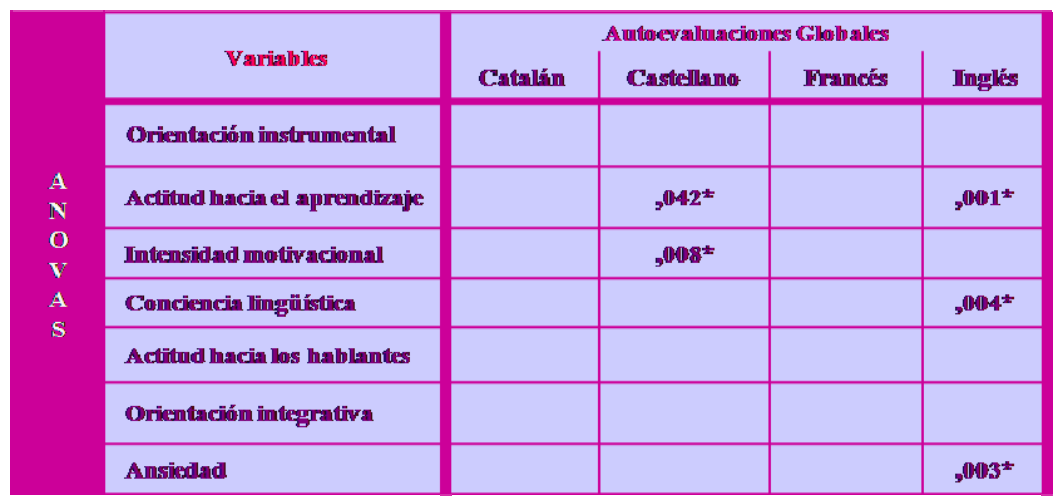

Fig.4: Anovas por Autoevaluaciones.

Estos resultados coinciden con el metanálisis realizado por Gardner y sus asociados (Masgoret y Gardner 2003) en el que las variables actitudes hacia el aprendizaje, el motivo integrativo, la motivación, la orientación integrativa y la orientación instrumental están relacionadas positivamente con la adquisición positiva de la lengua y las autoevaluaciones. De igual manera, diversos autores (Bernaus, Masgoret, Gardner y Reyes 2004; y Coleman 1995a) confirman cómo los estudiantes más motivados evalúan mejor su inglés en comparación con los estudiantes menos motivados. Lasagabaster (2003) explica cómo los estudiantes universitarios vascos, que viven en contextos preferentemente castellano parlantes, con una competencia elevada en lengua castellana, y conocimientos de otras lenguas además del eusquera, castellano e inglés, son los que manifiestan unas actitudes más positivas hacia el castellano. En cambio, en contextos vasco-parlantes, donde hay un mayor número de estudiantes con el eusquera como L1, que no cuentan con una competencia lingüística de castellano alta, las actitudes hacia la lengua castellana son negativas. Dadas sus actitudes hacia el inglés como LE, Lasagabaster (2003) corrobora que los estudiantes que adquieren los niveles alto y más alto son los que demuestran las mejores actitudes hacia la lengua. Otro autor que confirma con sus resultados la relación establecida entre el grado de motivación y el rendimiento 
académico es Madrid (1999); no obstante, según el autor (1999) en algunos casos, esta correlación no se cumple, y nos encontramos con unos resultados académicos bajos, y en cambio la motivación se mantiene alta. Para Madrid (1999), esto se explica por una presión social que vincula la LE a una necesidad profesional en el futuro.

Pavón (2001) difiere de estos resultados y afirma que la actitud no tiene una influencia directa en el aprendizaje sino que es la motivación (como resultado visible de las actitudes de los estudiantes) la que sí ejerce una influencia determinante, ya que, como mínimo en su estudio (con unas circunstancias muy definidas y unos sujetos muy particulares) no se ha podido demostrar que exista una relación estrecha entre lo que hemos denominado actitud y la competencia lingüística. Contrariamente a Pavón (2001) nuestros resultados sí que avalan la existencia de una vinculación muy estrecha entre las motivaciones y las actitudes con el aprendizaje de lenguas.

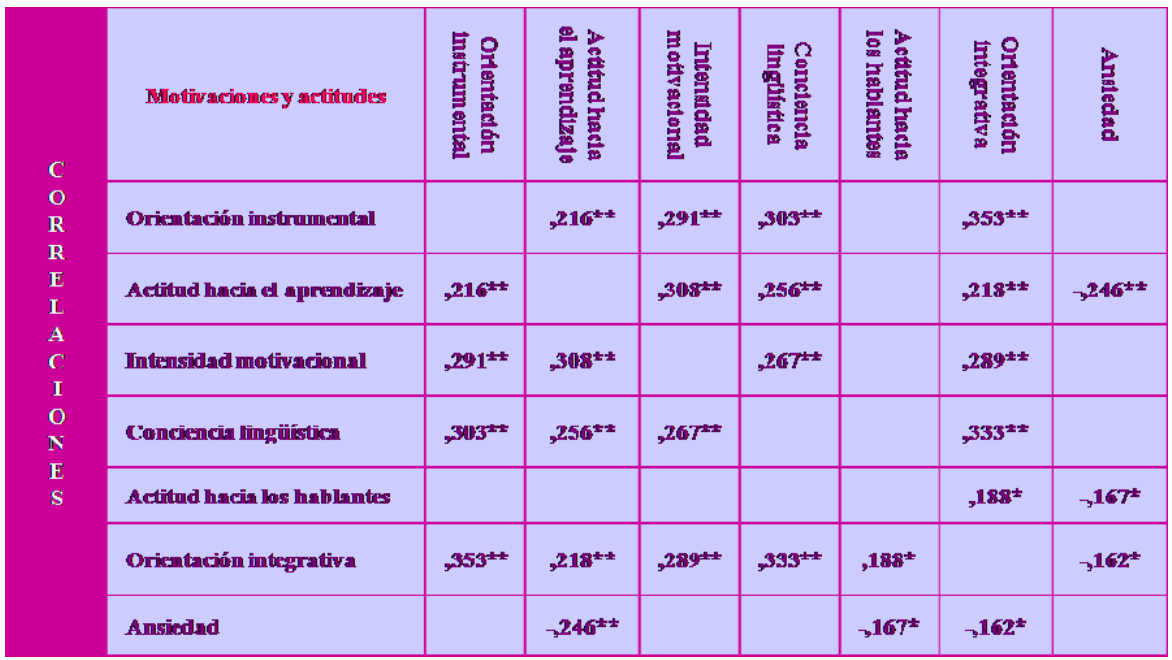

Fig. 5: Correlaciones en Motivaciones y Actitudes.

HIPÓTESIS 2: Los estudiantes de la Especialidad de Maestro en Lenguas Extranjeras (inglés) alcanzarán grados de motivación y actitudes más altos en el aprendizaje de lenguas, que los estudiantes de Enfermería y Fisioterapia.

Para validar o rechazar la hipótesis planteada, se han hecho análisis de la variancia. Los resultados demuestran que, efectivamente, los alumnos de Ciencias de la Educación disponen de una orientación instrumental, una orientación integrativa, una intensidad motivacional y una conciencia lingüística hacia las lenguas más marcadamente alta que los de Enfermería y Fisioterapia. No obstante, también manifiestan un grado de ansiedad más elevado que los estudiantes del ámbito sanitario. Todos estos resultados pueden ser fruto, quizá, de la orientación profesional escogida por los primeros. Sin duda, estos resultados avalan la hipótesis propuesta. Se puede afirmar, pues, que los estudiantes de la Especialidad de Maestro en Lenguas Extranjeras (Inglés), alcanzan grados de motivación y actitudes más altos en el 
aprendizaje de lenguas, que los estudiantes de Enfermería y Fisioterapia (ver cuadro Fig.3: Anovas por Escuelas).

Si comparamos los resultados obtenidos en nuestra investigación con los de estudios similares vemos que Coleman $(1998 ; 2002)$ reconoce que para los estudiantes del Reino Unido los objetivos prácticos corren paralelos al hecho de disfrutar de la lengua misma, y a unos sentimientos positivos hacia la lengua meta de la comunidad. Asimismo ocurre con los estudiantes procedentes de Ciencias de la Educación que cuentan tanto con una orientación instrumental como con una orientación integrativa elevadas; además los mismos manifiestan una intensidad motivacional y una conciencia lingüística hacia las lenguas más alta que los de Enfermería y Fisioterapia.

Tal como hemos dicho anteriormente, los estudiantes del ámbito educativo, que disponen de una orientación instrumental y una orientación integrativa más marcadamente alta, son justamente los que se autoevalúan mejor en cuanto a su competencia lingüística en catalán, francés e inglés, si los comparamos con los del ámbito sanitario. Por tanto, ambas orientaciones contribuyen a mejorar la competencia lingüística y a obtener mejores resultados. Martyn (2000) corrobora este hecho cuando explica que los aprendices de su estudio son generalmente adultos motivados, dado que el curso se centra en sus necesidades profesionales. La autora (2000) explica cómo los alumnos se esfuerzan por conseguir unos resultados excelentes, no tan solo para alcanzar los requerimientos del curso, sino también por provocar un impacto positivo, tanto personal como profesional, en su comunidad. Los motivos argumentados por Martyn (2000) son a la vez instrumentales e integrativos. A pesar de ello, parece que la orientación integrativa juega un papel determinante. De hecho, esto se corresponde con los resultados conseguidos por los futuros maestros; éstos demuestran una mayor orientación integrativa que corre pareja a una mejor autoevaluación en catalán, francés e inglés, respecto a los futuros enfermeros y fisioterapeutas.

A partir de los resultados de Coleman (1995a; 1998; 2002) los estudiantes que aprenden una lengua dentro de una titulación de ámbito científico muestran un porcentaje más elevado de orientación instrumental. Para el alumnado del ámbito educativo de nuestro trabajo, una buena competencia en la lengua inglesa está fuertemente ligada a las necesidades lingüísticas que identifican su situación profesional como futuros profesores de lenguas extranjeras inglés en la enseñanza primaria. Para los estudiantes de Enfermería y Fisioterapia existe una orientación profesional, en el sentido de que tal y como dice Coleman (1995) en su investigación, viene dada por el reconocimiento de que se trata de una lengua internacional, una lengua franca. Sin embargo, cabe decir que Lepetit y Cichock (1990) hablan de cómo las relaciones de tipo instrumental pueden aparecer como totalmente secundarias en ciertos grupos. Los autores (1990) atribuyen esta actitud al hecho de que probablemente las personas de la muestra son en su mayoría jóvenes adultos que acaban de entrar en la universidad; en otras palabras, o bien, no están integrados en el ámbito profesional, o bien todavía no tienen una idea precisa de cuál será su futuro profesional. Tal argumento puede servir también para explicar el porqué la orientación instrumental hacia la lengua extranjera sería secundaria para los futuros enfermeros o fisioterapeutas. Lo sería en el sentido de que la gran mayoría de los alumnos citados no se plantea su carrera profesional en un ámbito de proyección internacional, sino más bien local o nacional. Según Norris (2001) el rechazo, por unas razones instrumentales fuertes, en el estudio del inglés puede indicar que la lengua considerada como la más adecuada para una comunicación verbal diaria y habitual, es otra. De esta manera 
resulta razonable suponer que la mayoría de alumnos de Enfermería y Fisioterapia no ven el inglés como imprescindible para su futuro profesional y, por tanto, su motivación no muestra correlaciones altas con el aprendizaje de la lengua.

La segunda hipótesis queda pues avalada.

\section{Conclusiones Finales}

El objetivo de este estudio, como ya se ha mencionado, ha sido el de ver la influencia que los factores individuales pueden tener en el aprendizaje de lenguas en estudiantes universitarios.

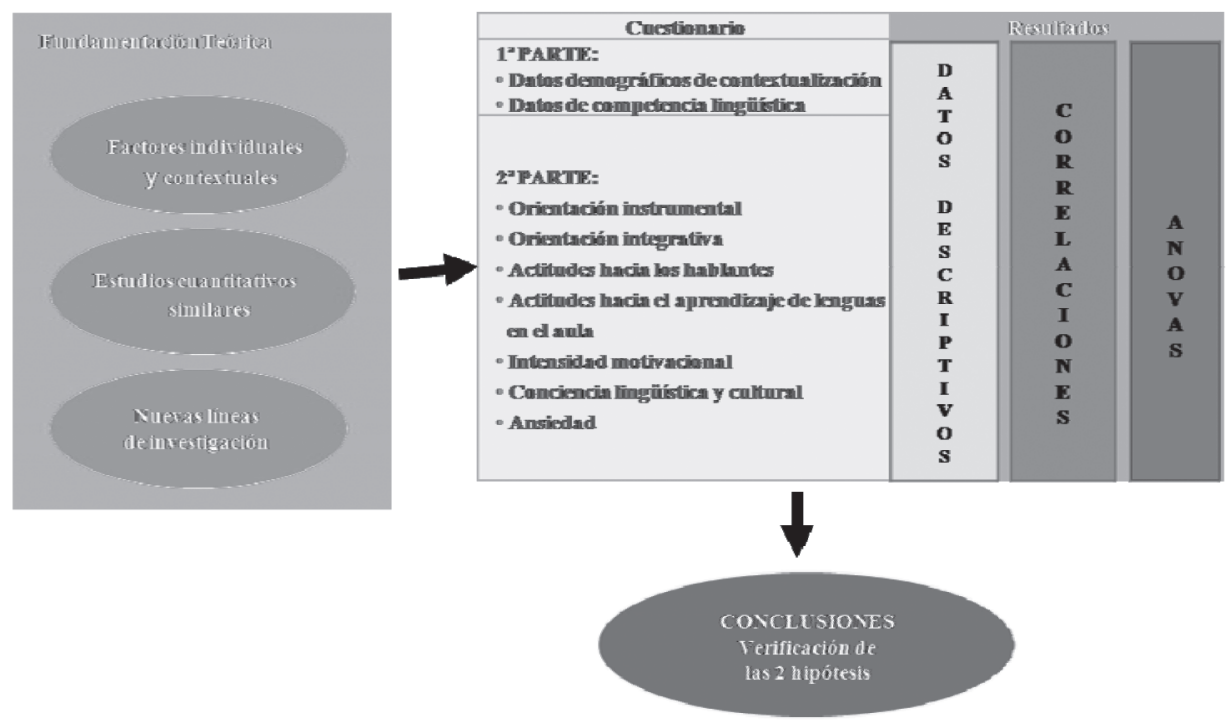

Fig. 6: Conclusiones Finales.

En el apartado de Resultados, hemos verificado dos hipótesis, comparando los resultados obtenidos con los de investigaciones semejantes a la nuestra. En consecuencia, por todo lo que se ha comentado anteriormente, podemos afirmar con satisfacción que este estudio es una nueva aportación a la investigación en docencia universitaria, especialmente en el ámbito de lenguas. Sus aportaciones principales han sido primero la de hacer extensivo este tipo de investigación al mundo universitario en Cataluña. En segundo lugar, el ser una investigación multilingüe, puesto que ha relacionado los factores individuales con las cuatro lenguas de instrucción: catalán, castellano, inglés y francés.

En cuanto a posteriores investigaciones, por un lado, proponemos analizar la motivación como algo cambiante que evoluciona a lo largo del aprendizaje (Dörnyei 2003) y no analizarla puntualmente como en este estudio. Para tal estudio se debería pasar un cuestionario a 
principio y a final de curso, y ver la evolución de los alumnos (Bernaus y Gardner, 2004, inédito).

Con vistas al futuro, considerando que el contexto educativo de los aprendices va camino de ser cada día más multilingüe y multicultural (Frank 2000, Duff 2002, Levine 2003, Lasagabaster 2003, y Bernaus, Masgoret, Gardner, y Reyes 2004), los futuros trabajos de investigación tendrían que tener en cuenta esta realidad, prestando atención a un contexto social, cada vez más complejo y cambiante, que da paso a una realidad multilingüe y multicultural

Antes de acabar, queremos presentar nuestro agradecimiento a los profesores de la Escuela Universitaria de Enfermería y Fisioterapia Blanquerna, URL, que introdujeron los datos en el programa informático SPSS; a los profesores y compañeros de la Facultad de Ciencias de la Educación de la UAB, que aceptaron que este cuestionario se pasara a sus estudiantes; a los estudiantes que lo respondieron; y por descontado, la dirección de este estudio, a la Dra. Mercè Bernaus.

\section{RefERencias Bibliográficas}

Alonso Tapia, J. (1999). "Motivación y aprendizaje en la Enseñanza Secundaria", en C. Coll. (Coord.) Psicología de la Instrucción: la enseñanza y el aprendizaje en la educación secundaria, Barcelona, ICE - Horsori, cap. IV, p. 105-140.

Bernaus, M., Masgoret, A.-M., Gardner, R.C., y Reyes, E (2004). "Motivation and Attitudes toward Learning Languages in Multicultural Classrooms", en International Journal of Multilingualism, Multilingual Matters.

Bernaus, M. y Gardner, R.C. (2004). The applicability of the Attitude/Motivation Test Battery to EFL students in Spain. Unpublished manusript, University of Western Ontario.

Coleman, J. A. (1995a). Progress, proficiency and motivation among University Language Learners, Dublin, Centre for Language and Communication Studies (Trinity College Dublin), Occasional Paper núm. 40.

Coleman, J. (1998; 2002). "Motivation among university language students in the UK and in continental Europe", en Research Forum Papers, Centre for Information on Language Teaching and Research, CILT web site: http://www.cilt.org.uk 23/5/02

Costa, P. T. y McCrae, R. R. (1988). "From catalog to classification: Murray's needs and the fivefactor model", en Journal of Personality and Social Psychology, 55: 258-265.

Dörnyei, Z. (2000). Teaching and researching motivation, Harlow. UK: Addison Wesley Longman.

Dörnyei, Z. (2001a). Motivational Strategies in the Language Classroom. Cambridge: Cambridge University Press.

Dörnyei, Z. (2002). "The motivational basis of language learning tasks", en P. Robinson, (ed.). Individual Differences in Second Language Acquisition, Amsterdam, John Benjamins, 137-158.

Dörnyei, Z. (2003). "Attitudes, Orientations, and Motivation in Language Learning: Advances in Theory, Research, and Applications", en Attitudes, Orientations, and Motivation in Language Learning.

Duff, P. A., Wong, P. y Early, M. (2002). "Learning Language for Work and Life: The Linguistic Socialization of Immigrant Canadians Seeking Careers in Healthcare", en The Modern Language Journal, 86: iii

Ely, C. M. (1986). "Language learning motivation: a descriptive and causal analysis", en The Modern Language Journal, 70, 1: 28-35. 
Frank, R. A. (2000). "Medical communication: non-native English speaking patients and native English speaking professionals", en English for Specific Purposes, 19: 31-62.

Gardner, R. (1979). "Social psychological aspects of second language acquisition", en H. Giles, y ST. R. Clair (ed.): Language and social psychology, Oxford, UK, Basil Blackwell, 193220.

Gardner, R. (1985). Social Psychology and Second Language Learning, The Roles of Attitudes and Motivation. London: Edward Arnold.

Gardner, R.. (2003). "Integrative motivation, induced anxiety, and language learning in a controlled environment", en Studies in Second Language Acquisition, 14: 197-214.

Knowles, M. S., Holton III, E. y Swanson, R. A. (2001). Andragogía: El aprendizaje de los adultos, Mèxic. Oxford: Oxford University Press.

Lasagabaster, D. (2003). Trilingüismo en la Enseñanza: Actitudes hacia la lengua minoritaria, la mayoritaria y la extranjera. Lleida: Editorial Milenio.

Lepetit, D. y Cichock, W. (1990). "Aspirations langagières: Négociation et apprentissage du français", en The Canadian Modern Language Review/La Revue canadienne des langues vivantes, 46, 2.

Lepetit, D. y Cichock, W. (2002). "Teaching Languages to Future Health Professionals: A Needs Assessment Study", en The Modern Language Journal, 86: iii.

Levine, G. S. (2003). "Student and Instructor Beliefs and Attitudes about Target Language Use, First Language Use, and Anxiety: Report of a Questionnaire Study", en The Modern Language Journal, 87: iii.

Lightbown, P. y Spada, N. (1993). How languages are learned. Oxford: Oxford University Press.

Linde, A. (2001). "Investigating university students' attitudes towards assessment", en Actas del 25 Congreso AEDEAN (Asociación Española de Estudios Anglo-norteamericanos), Departamento de Filología Inglesa, Universidad de Granada, 13, 14 y 15 de diciembre, Granada 2001.

Madrid, D.(1999). La investigación de los Factores Motivacionales en el Aula de Idiomas. Granada: Grupo Editorial Universitario.

Martyn, E. (2000). "Syllabus negotiation in a school of nursing", en M. Breen \& A. Littlejohn, (ed.) Classroom Decision-Making (Negotiation and process syllabuses in practice), Cambridge: Cambridge University Press, p.150-162.

Masgoret, A. M., Bernaus, M. y Gardner R. C. (2000). "A Study of Cross-Cultural Adaptation by English-Speaking Sojourners in Spain", en Foreign Language Annals, vol. 33, 5: 548558.

Masgoret, A. M. y Gardner, R. C. (2003). "Attitudes, motivation and Second Language Learning: A meta-analysis of studies conducted by Gardner and Associates", en Language Learning, 53, 1: 123-163.

Norris, J. (2001). "Motivation as a Contributing Factor in Second Language Acquisition", The Internet TESL Journal, vol. VII, núm. 6. June 2001. Web site: http://teslj.org/; http:// iteslj.org/Articles/Norris-Motivation.html

Oxford, R. (1990). Language Learning Strategies: What Every Teacher Should Know. New York: Newbury House.

Oxford, R. (1996). Language Learning Motivation: Pathway to the new century. Hawaii: University of Hawaii.

Pavón, V. (2001). "Un estudio sobre la influencia del componente afectivo actitud en la competencia de aprendices adultos de inglés", en C.Muñoz (2001). Trabajos en lingüística aplicada, Barcelona: Aesla. 
Ramage, K. (1990). "Motivational factors and persistence in Foreign Language Study", en Language Learning, 40, 2: 189-219.

Saravia, E. (2002). Reflexions per a l'elaboració d'un qüestionari capaç d'avaluar la influència dels factors individuals en l'aprenentatge de llengües, Trabajo de Investigación, Doctorat en Didàctica de la Llengua, de la Literatura i de les Ciències Socials, FCE-UAB, dirigido por la Dra. Mercè Bernaus.

Saravia, E. (2004). La Influència dels factors individuals en l'aprenentatge de llengües en estudiants universitàris, Tesis Doctoral no publicada, Barcelona, Universidad Autònoma de Barcelona, dirigida por la Dra. Mercè Bernaus.

Velasco, C. y Bernaus, M. (1999). "Los proyectos en el aula de lengua: una herramienta para la motivación", en Aula de Innovación Educativa, Barcelona, núm. 87: 39-42.

Yalden, J. (1987). Principles of Course Design for Language Teaching. Cambridge: Cambridge University Press. 
APÉNDICE: Apartado de motivación y actitudes - sección 2

\section{A) QÜESTIONARI INFERMERIA I FISIOTERÀPIA}

2a part

El propòsit del qüestionari és conèixer les teves opinions sobre les llengües i el seu estudi.

Si us plau, valora els enunciats següents segons l'escala de valoració proposada de 1'1 al 5.

Encercla el número que creguis més adient.

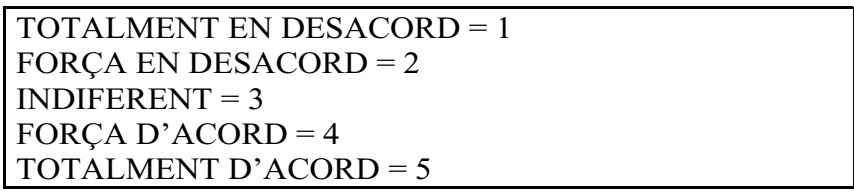

Enunciats:

El que aprendré en aquesta assignatura em serà útil a la meva feina.

$\begin{array}{lllllll}\text { TOTALMENT EN } & 1 & 2 & 3 & 4 & 5 & \text { TOTALMENT } \\ \text { DESACORD } & & & & & & \text { D'ACORD }\end{array}$

L'assignatura de català / francès / anglès serà fàcil d'aprovar.

$\begin{array}{lllllll}\text { TOTALMENT EN } & 1 & 2 & 3 & 4 & 5 & \begin{array}{l}\text { TOTALMENT } \\ \text { D'ACORD }\end{array}\end{array}$

Quan he de parlar en català / francès / anglès davant els meus companys de classe estic tranquil.

$\begin{array}{lllllll}\begin{array}{l}\text { TOTALMENT EN } \\ \text { DESACORD }\end{array} & 1 & 2 & 3 & 4 & 5 & \begin{array}{l}\text { TOTALMENT } \\ \text { D'ACORD }\end{array}\end{array}$

Faig un esforç per escriure bé en català/francès / anglès.

$\begin{array}{llllllll}\text { TOTALMENT EN } & 1 & 2 & 3 & 4 & 5 & \text { TOTALMENT } \\ \text { DESACORD } & & & & & & \text { D'ACORD }\end{array}$

Conèixer una llengua em permet conèixer una cultura.

$\begin{array}{lllllll}\text { TOTALMENT EN } & 1 & 2 & 3 & 4 & 5 & \begin{array}{l}\text { TOTALMENT } \\ \text { D'ACORD }\end{array}\end{array}$

Els catalans / francesos / anglesos em cauen bé.

$\begin{array}{llllllll}\text { TOTALMENT EN } & 1 & 2 & 3 & 4 & 5 & \begin{array}{l}\text { TOTALMENT } \\ \text { DESACORD }\end{array} \\ \text { D'ACORD }\end{array}$

Aprendre llengües em fa sentir membre actiu de l'Europa comunitària.
TOTALMENT EN
23
45
TOTALMENT
DESACORD
D'ACORD

Si domino el català / el francès / l'anglès seré més valorat.

$\begin{array}{lllllll}\begin{array}{l}\text { TOTALMENT EN } \\ \text { DESACORD }\end{array} & 1 & 2 & 3 & 4 & 5 & \begin{array}{l}\text { TOTALMENT } \\ \text { D'ACORD }\end{array}\end{array}$


Sóc de ciències i les lletres mai no m'han interessat.
TOTALMENT EN
1
2
3
4
DESACORD
5
TOTALMENT
D'ACORD

Envio correus electrònics en català / francès / anglès sense que em preocupi fer faltes d'ortografia.
TOTALMENT EN 1
12
3
$4 \quad 5$
TOTALMENT
DESACORD
D'ACORD

Si no hagués pogut fer l'assignatura de català/ francès / d'anglès a l'EUIFB, aniria a classes de català/ francès / anglès fora de la universitat.
TOTALMENT EN
23
$4 \quad 5$
TOTALMENT
DESACORD
D'ACORD

El fet de parlar diverses llengües m'enriqueix de forma personal i professional.
TOTALMENT EN
1
23
4
5
TOTALMENT
DESACORD
D'ACORD

Els catalans / francesos / anglesos són molt creguts.
TOTALMENT EN
12
3
4
5
TOTALMENT
DESACORD
D'ACORD

El coneixement de llengües em permet fer nous amics.
TOTALMENT EN
1
2
3
4
DESACORD
5 TOTALMENT
D'ACORD

La classe de llengua em pot ajudar a aprovar exàmens.
TOTALMENT EN
1
2
3
4
DESACORD
5
TOTALMENT
D'ACORD

He triat aquesta assignatura perquè no en quedava cap altra.
TOTALMENT EN
1
2
34
5
DESACORD
TOTALMENT
D'ACORD

Em preocupa l'exigència del professor a l'hora de corregir un examen de català / francès / anglès.
TOTALMENT EN 1
12
3
$4 \quad 5$
5 TOTALMENT
DESACORD
D'ACORD

Si no entenc alguna cosa a classe de català / francès / d'anglès pregunto immediatament al professor o als companys.
TOTALMENT EN 1
DESACORD
23
$4 \quad 5$
TOTALMENT
D'ACORD

La diversitat dialectal i lingüística no són un obstacle sinó una riquesa.
TOTALMENT EN
1
2
3
4
5
TOTALMENT
DESACORD
D'ACORD 
Els catalans / francesos / anglesos són acollidors.

$\begin{array}{lllllll}\begin{array}{l}\text { TOTALMENT EN } \\ \text { DESACORD }\end{array} & 1 & 2 & 3 & 4 & 5 & \begin{array}{l}\text { TOTALMENT } \\ \text { D'ACORD }\end{array}\end{array}$

Els costums dels castellans són molt diferents dels nostres. / Els costums dels francesos són molt diferents dels nostres. / Els costums dels anglesos són molt diferents dels nostres.

$\begin{array}{lllllll}\begin{array}{l}\text { TOTALMENT EN } \\ \text { DESACORD }\end{array} & 1 & 2 & 3 & 4 & 5 & \begin{array}{l}\text { TOTALMENT } \\ \text { D'ACORD }\end{array}\end{array}$

Aquesta assignatura em pot ajudar a escriure un article per una revista científica, a participar en congressos internacionals, etc.

$\begin{array}{lllllll}\begin{array}{l}\text { TOTALMENT EN } \\ \text { DESACORD }\end{array} & 1 & 2 & 3 & 4 & 5 & \begin{array}{l}\text { TOTALMENT } \\ \text { D'ACORD }\end{array}\end{array}$

Els professors de català / francès / anglès sempre m'han resultat avorrits.

$\begin{array}{lllllll}\begin{array}{l}\text { TOTALMENT EN } \\ \text { DESACORD }\end{array} & 1 & 2 & 3 & 4 & 5 & \begin{array}{l}\text { TOTALMENT } \\ \text { D'ACORD }\end{array}\end{array}$

Quan he de fer treballs en grup em neguiteja pensar que no em puc expressar tan bé com els altres en català / francès / anglès.

$\begin{array}{lllllll}\begin{array}{l}\text { TOTALMENT EN } \\ \text { DESACORD }\end{array} & 1 & 2 & 3 & 4 & 5 & \begin{array}{l}\text { TOTALMENT } \\ \text { D'ACORD }\end{array}\end{array}$

Els treballs de català / francès / anglès, els faig sempre a última hora.

$\begin{array}{lllllll}\text { TOTALMENT EN } & 1 & 2 & 3 & 4 & 5 & \text { TOTALMENT } \\ \text { DESACORD } & & & & & & \text { D'ACORD }\end{array}$

Aprendre una llengua minoritària és una pèrdua de temps.

$\begin{array}{lllllll}\text { TOTALMENT EN } & 1 & 2 & 3 & 4 & 5 & \text { TOTALMENT } \\ \text { DESACORD } & & & & & & \text { D'ACORD }\end{array}$

Els catalans / francesos / anglesos són reservats.

$\begin{array}{lllllll}\text { TOTALMENT EN } & 1 & 2 & 3 & 4 & 5 & \begin{array}{l}\text { TOTALMENT } \\ \text { DESACORD }\end{array}\end{array}$

No m'agradaria viure en un país de llengua anglesa / francesa. / No m'agradaria quedar-me a viure per sempre a Catalunya.
TOTALMENT EN
23
4
5
TOTALMENT
DESACORD
D'ACORD

Un bon coneixement del català / del francès / de l'anglès em pot obrir camins.
TOTALMENT EN
1
23
45
TOTALMENT DESACORD
D'ACORD

Les classes de català / francès / anglès que he tingut abans de ser estudiant a l'EUIF m'han agradat.

$\begin{array}{lllllll}\begin{array}{l}\text { TOTALMENT EN } \\ \text { DESACORD }\end{array} & 1 & 2 & 3 & 4 & 5 & \begin{array}{l}\text { TOTALMENT } \\ \text { D'ACORD }\end{array}\end{array}$


Quan faig exàmens orals en català / francès / anglès estic molt tranquil.

$\begin{array}{lllllll}\text { TOTALMENT EN } & 1 & 2 & 3 & 4 & 5 & \begin{array}{l}\text { TOTALMENT } \\ \text { D'ACORD }\end{array} \\ \text { DESACORD } & & & & & & \end{array}$

Quan em tornen els treballs corregits els repasso sempre.

$\begin{array}{lllllll}\text { TOTALMENT EN } & 1 & 2 & 3 & 4 & 5 & \begin{array}{l}\text { TOTALMENT } \\ \text { D'ACORD }\end{array} \\ \text { DESACORD } & & & & & & \end{array}$

Si et pots comunicar en una segona llengua, no cal aprendre la llengua del lloc on vius.

$\begin{array}{lllllll}\text { TOTALMENT EN } & 1 & 2 & 3 & 4 & 5 & \begin{array}{l}\text { TOTALMENT } \\ \text { D'ACORD }\end{array}\end{array}$

Els catalans / francesos / anglesos són sincers.

\begin{tabular}{l}
$\begin{array}{l}\text { TOTALMENT EN } \\
\text { DESACORD }\end{array}$ \\
\hline
\end{tabular}

Penso que parlo millor en català / francès / anglès que els meus companys.

$\begin{array}{lllllll}\begin{array}{l}\text { TOTALMENT EN } \\ \text { DESACORD }\end{array} & 1 & 2 & 3 & 4 & 5 & \begin{array}{l}\text { TOTALMENT } \\ \text { D'ACORD }\end{array}\end{array}$

Considerant el que faig a classe i fora de classe per aprendre català / francès / anglès, puc dir que faig el mínim per aprovar.

$\begin{array}{lllllll}\begin{array}{l}\text { TOTALMENT EN } \\ \text { DESACORD }\end{array} & 1 & 2 & 3 & 4 & 5 & \begin{array}{l}\text { TOTALMENT } \\ \text { D'ACORD }\end{array}\end{array}$

Espero amb moltes ganes la classe de català / francès / anglès.

$\begin{array}{lllllll}\begin{array}{l}\text { TOTALMENT EN } \\ \text { DESACORD }\end{array} & 1 & 2 & 3 & 4 & 5 & \begin{array}{l}\text { TOTALMENT } \\ \text { D'ACORD }\end{array}\end{array}$

Em fa vergonya no pronunciar bé el català / francès / 1'anglès.

$\begin{array}{lllllll}\text { TOTALMENT EN } & 1 & 2 & 3 & 4 & 5 & \begin{array}{l}\text { TOTALMENT } \\ \text { DESACORD }\end{array} \\ & & & & & & \text { D'ACORD }\end{array}$

Evito les ocasions per practicar el català / francès / anglès fora de la classe.

$\begin{array}{lllllll}\text { TOTALMENT EN } & 1 & 2 & 3 & 4 & 5 & \text { TOTALMENT } \\ \text { DESACORD } & & & & & & \text { D'ACORD }\end{array}$

Si amb l'anglès es va a tot arreu, no cal estudiar altres llengües.

$\begin{array}{lllllll}\text { TOTALMENT EN } & 1 & 2 & 3 & 4 & 5 & \text { TOTALMENT } \\ \text { DESACORD } & & & & & & \text { D'ACORD }\end{array}$

Els catalans / francesos / anglesos són gent en qui no pots confiar.

$\begin{array}{lllllll}\text { TOTALMENT EN } & 1 & 2 & 3 & 4 & 5 & \begin{array}{l}\text { TOTALMENT } \\ \text { D'ACORD }\end{array}\end{array}$

Em fa por no entendre el que diu el professor a classe de català / francès / anglès.

$\begin{array}{lllllll}\begin{array}{l}\text { TOTALMENT EN } \\ \text { DESACORD }\end{array} & 1 & 2 & 3 & 4 & 5 & \begin{array}{l}\text { TOTALMENT } \\ \text { D'ACORD }\end{array}\end{array}$


Considerant la meva participació en els treballs en grup, penso que faig el mínim esforç.
TOTALMENT EN
2
3
45
DESACORD
TOTALMENT
D'ACORD

\section{B) QÜESTIONARI CIÈNCIES DE L'EDUCACIÓ}

\section{2a part}

El propòsit del qüestionari és conèixer les teves opinions sobre les llengües i el seu estudi. Si us plau, valora els enunciats següents segons l'escala de valoració proposada de 1'1 al 5. Encercla el número que creguis més adient.

$$
\begin{aligned}
& \text { TOTALMENT EN DESACORD }=1 \\
& \text { FORÇA EN DESACORD }=2 \\
& \text { INDIFERENT }=3 \\
& \text { FORÇA D'ACORD }=4 \\
& \text { TOTALMENT D'ACORD }=5
\end{aligned}
$$

15. El que aprendré en aquesta assignatura em serà útil a la meva feina.

$\begin{array}{lllllll}\begin{array}{l}\text { TOTALMENT EN } \\ \text { DESACORD }\end{array} & 1 & 2 & 3 & 4 & 5 & \begin{array}{l}\text { TOTALMENT } \\ \text { D'ACORD }\end{array}\end{array}$

16. Les assignatures de llengües estrangeres seran fàcils d'aprovar.

$\begin{array}{lllllll}\text { TOTALMENT EN } & 1 & 2 & 3 & 4 & 5 & \text { TOTALMENT } \\ \text { DESACORD } & & & & & & \text { D'ACORD }\end{array}$

17. Quan he de parlar en anglès davant els meus companys de classe estic tranquil.

$\begin{array}{lllllll}\text { TOTALMENT EN } & 1 & 2 & 3 & 4 & 5 & \begin{array}{l}\text { TOTALMENT } \\ \text { DESACORD }\end{array} \\ \text { D'ACORD }\end{array}$

18. Faig un esforç per escriure bé en anglès.

$\begin{array}{llllllll}\text { TOTALMENT EN } & 1 & 2 & 3 & 4 & 5 & \begin{array}{l}\text { TOTALMENT } \\ \text { DESACORD }\end{array} \\ \text { D'ACORD }\end{array}$

19. Conèixer una llengua em permet conèixer una cultura.

$\begin{array}{llllllll}\text { TOTALMENT EN } & 1 & 2 & 3 & 4 & 5 & \begin{array}{l}\text { TOTALMENT } \\ \text { DEACORD }\end{array}\end{array}$

20. Els anglesos em cauen bé.

$\begin{array}{lllllll}\begin{array}{l}\text { TOTALMENT EN } \\ \text { DESACORD }\end{array} & 1 & 2 & 3 & 4 & 5 & \begin{array}{l}\text { TOTALMENT } \\ \text { D'ACORD }\end{array}\end{array}$

21. Aprendre llengües em fa sentir membre actiu de l'Europa comunitària.
TOTALMENT EN
1
23
$3 \quad 4 \quad 5$
5 TOTALMENT
DESACORD
D'ACORD

22. Si domino l'anglès seré més valorat. 
34. Els anglesos són acollidors.

$\begin{array}{lllllll}\begin{array}{l}\text { TOTALMENT EN } \\ \text { DESACORD }\end{array} & 1 & 2 & 3 & 4 & 5 & \begin{array}{l}\text { TOTALMENT } \\ \text { D'ACORD }\end{array}\end{array}$

35. Els costums dels anglesos són molt diferents dels nostres.

$\begin{array}{lllllll}\begin{array}{l}\text { TOTALMENT EN } \\ \text { DESACORD }\end{array} & 1 & 2 & 3 & 4 & 5 & \begin{array}{l}\text { TOTALMENT } \\ \text { D'ACORD }\end{array}\end{array}$

36. Saber força anglès em pot ajudar a elaborar materials, a escriure articles, a participar en congressos internacionals, etc.

$\begin{array}{lllllll}\begin{array}{l}\text { TOTALMENT EN } \\ \text { DESACORD }\end{array} & 1 & 2 & 3 & 4 & 5 & \begin{array}{l}\text { TOTALMENT } \\ \text { D'ACORD }\end{array}\end{array}$

37. Els professors d'anglès sempre m'han resultat avorrits.

$\begin{array}{lllllll}\text { TOTALMENT EN } & 1 & 2 & 3 & 4 & 5 & \begin{array}{l}\text { TOTALMENT } \\ \text { D'ACORD }\end{array}\end{array}$

38. Quan he de fer treballs en grup em neguiteja pensar que no em puc expressar tan bé com els altres en anglès.

$\begin{array}{lllllll}\text { TOTALMENT EN } & 1 & 2 & 3 & 4 & 5 & \text { TOTALMENT }\end{array}$

DESACORD

D'ACORD

39. Els treballs d'anglès, els faig sempre a última hora.

$\begin{array}{lllllll}\text { TOTALMENT EN } & 1 & 2 & 3 & 4 & 5 & \begin{array}{l}\text { TOTALMENT } \\ \text { D'ACORD }\end{array} \\ \text { DESACORD } & & & & & & \end{array}$

40. Aprendre una llengua minoritària és una pèrdua de temps.

$\begin{array}{lllllll}\text { TOTALMENT EN } & 1 & 2 & 3 & 4 & 5 & \begin{array}{l}\text { TOTALMENT } \\ \text { D'ACORD }\end{array} \\ \text { DESACORD } & & & & & & \end{array}$

41. Els anglesos són reservats.

$\begin{array}{lllllll}\begin{array}{l}\text { TOTALMENT EN } \\ \text { DESACORD }\end{array} & 1 & 2 & 3 & 4 & 5 & \begin{array}{l}\text { TOTALMENT } \\ \text { D'ACORD }\end{array}\end{array}$

42. No m'agradaria viure en un país de llengua anglesa.

$\begin{array}{lllllll}\text { TOTALMENT EN } & 1 & 2 & 3 & 4 & 5 & \begin{array}{l}\text { TOTALMENT } \\ \text { D'ACORD }\end{array}\end{array}$

43. Un bon coneixement de l'anglès em pot obrir portes a l'Europa Comunitària.

$\begin{array}{lllllll}\text { TOTALMENT EN } & 1 & 2 & 3 & 4 & 5 & \text { TOTALMENT } \\ \text { DESACORD } & & & & & & \text { D'ACORD }\end{array}$

44. Les classes d'anglès que he tingut abans de ser estudiant a la U.A.B. m'han agradat.

$\begin{array}{llllllll}\begin{array}{l}\text { TOTALMENT EN } \\ \text { DESACORD }\end{array} & 1 & 2 & 3 & 4 & 5 & \begin{array}{l}\text { TOTALMENT } \\ \text { D'ACORD }\end{array}\end{array}$

45. Quan faig exàmens orals en anglès estic molt tranquil.

$\begin{array}{llllllll}\text { TOTALMENT EN } & 1 & 2 & 3 & 4 & 5 & \text { TOTALMENT }\end{array}$ 
46. Quan em tornen els treballs corregits els repasso sempre.
TOTALMENT EN
2
34
45
DESACORD
D'ACORD

47. Si et pots comunicar en una segona llengua, no cal aprendre la llengua del lloc on vius.
TOTALMENT EN
1
23
4
5
TOTALMENT
DESACORD
D'ACORD

48. Els anglesos són sincers.

$\begin{array}{lllllll}\begin{array}{l}\text { TOTALMENT EN } \\ \text { DESACORD }\end{array} & 1 & 2 & 3 & 4 & 5 & \begin{array}{l}\text { TOTALMENT } \\ \text { D'ACORD }\end{array}\end{array}$

49. Penso que parlo millor en anglès que els meus companys.
TOTALMENT EN
1
2
345
5 TOTALMENT
DESACORD
D'ACORD

50. Considerant el que faig a classe i fora de classe per aprendre anglès, puc dir que faig el mínim per aprovar.
TOTALMENT EN
1
2
3
4
5
TOTALMENT
DESACORD
D'ACORD

Espero amb moltes ganes la classe d'anglès.

$\begin{array}{lllllll}\text { TOTALMENT EN } & 1 & 2 & 3 & 4 & 5 & \begin{array}{l}\text { TOTALMENT } \\ \text { DEACORD }\end{array}\end{array}$

52. Em fa vergonya no pronunciar bé 1'anglès.

$\begin{array}{lllllll}\begin{array}{l}\text { TOTALMENT EN } \\ \text { DESACORD }\end{array} & 1 & 2 & 3 & 4 & 5 & \begin{array}{l}\text { TOTALMENT } \\ \text { D'ACORD }\end{array}\end{array}$

53. Evito les ocasions per practicar l'anglès fora de la classe.

$\begin{array}{lllllll}\text { TOTALMENT EN } & 1 & 2 & 3 & 4 & 5 & \begin{array}{l}\text { TOTALMENT } \\ \text { D'ACORD }\end{array}\end{array}$

54. Si amb l'anglès es va a tot arreu, no cal estudiar altres llengües.
TOTALMENT EN
1
45
TOTALMENT
DESACORD

D'ACORD

55. Els anglesos són gent en qui no pots confiar.
TOTALMENT EN
12
34
5
TOTALMENT
DESACORD
D'ACORD

56. Em fa por no entendre el que diu el professor a classe d'anglès.
TOTALMENT EN
12
3
4
5
TOTALMENT
DESACORD
D'ACORD

57. Considerant la meva participació en els treballs en grup, penso que faig el mínim esforç
TOTALMENT EN
1
2
3
45
TOTALMENT
DESACORD
D'ACORD 